\title{
THE ANDERSON MODEL WITH MISSING SITES
}

\author{
CONSTANZA Rojas-Molina
}

Abstract. In the present note we show dynamical localization for an Anderson model with missing sites in a discrete setting at the bottom of the spectrum in arbitrary dimension $d$. In this model, the random potential is defined on a relatively dense subset of $\mathbb{Z}^{d}$, not necessarily periodic, i.e., a Delone set in $\mathbb{Z}^{d}$. To work in the lower band edge we need no further assumption on the geometric complexity of the Delone set. We use a spatial averaging argument by BourgainKenig to obtain a uniform Wegner estimate and an initial length scale estimate, which yields localization through the Multiscale Analysis for non ergodic models. This argument gives an explicit dependence on the maximal distance parameter of the Delone set for the Wegner estimate. We discuss the case of the upper spectral band edge and the arising need of imposing the (complexity) condition of strict uniform pattern frequency on the Delone set.

Mathematics subject classification (2010): 82B44 (Primary), 47B80, 47H40 (Secondary).

Keywords and phrases: Random Schrödinger operators, Wegner estimate, Delone-Anderson operators, dynamical localization, Delone dynamical systems.

\section{REFERENCES}

[1] M. Aizenman And S. A. Molchanov, Localization at large disorder and at extreme energies: an elementary derivation, Comm. Math. Phys., 157 (1993), 245-278.

[2] J. Bourgain AND C. Kenig, On localization in the continuous Anderson-Bernoulli model in higher dimension, Invent. Math., 161 (2005), 389-426.

[3] A. Boutet de Monvel, S. Naboko, P. Stollmann, And G. Stolz, Localization near fluctuation boundaries via fractional moments and applications, J. Anal. Math., 100 (2006), 83-116.

[4] Z. CAO AND A. Elgart, The weak localization for the alloy-type Anderson model on a cubic lattice, J. Stat. Phys., 148, 6 (2012), 1006-1039.

[5] J.-M. CombeS, P. D. Hislop, AND F. KLOPP, Hölder continuity of the integrated density of states for some random operatos at all energies, I.M.R.N., 4 (2003), 179-209.

[6] J.-M. Combes, P. Hislop, AND F. Klopp, An optimal Wegner estimate and its application to the global continuity of the IDS for random Schrödinger operators, Duke Math. J., 140, 3 (2007), 469498.

[7] A. Elgart And A. Klein, Ground state energy of trimmed discrete Schrödinger operators and localization for trimmed Anderson models, J. Spectr. Theory, to appear, arXiv:1301.5268, 2013.

[8] F. GERMINET, Recent advances about localization in continuum random Schrödinger operators with an extension to underlying Delone sets, pages 79-96, Mathematical results in quantum mechanics. World Sci. Publ., Hackensack, NJ, 2008.

[9] F. Germinet, P. D. Hislop, AND A. Klein, Localization for Schrödinger operators with Poisson random potential, JEMS, 9 (2007), 577-607.

[10] F. Germinet And A. Klein, Bootstrap multiscale analysis and localization in random media, Comm. Math. Phys., 222 (2001), 415-448.

[11] F. Germinet, P. MÜller, And C. Rojas-Molina, Ergodicity and dynamical localization for Delone-Anderson operators, In preparation.

[12] W. KIRSCH, An invitation to random Schrödinger operators, In Random Schrödinger operators, volume 25 of Panoramas et Synthèses. SMF, 2008. 
[13] A. KLEIN, Unique continuation principle for spectral projections of Schrödinger operators and optimal Wegner estimates for non-ergodic random Schrödinger operators, Commun. Math. Phys., 323 (2013), 1229-1246.

[14] A. KLEIN, Multiscale Analysis and Localization of random operators, In Random Schrödinger operators, volume 25 of Panoramas et Synthèses. SMF, 2008.

[15] W. KIRSCH AND I. VeSELIĆ, Existence of the density of states for one-dimensional alloy-type potentials with small support, Contemp. Math., 307 (2002), 171-176.

[16] D. LenZ And P. Stollmann, Delone dynamical systems and associated random operators, In J. M. Combes et al., editors, Proc. OAMP, Constanta 2001. Theta Foundation, 2003.

[17] D. Lenz And P. Stollmann, An ergodic theorem for delone dynamical systems and existence of the integrated density of states, J. Anal. Math., 97 (2005), 1-24.

[18] D. LenZ AND P. Stollmann, Generic sets in spaces of measures and generic singular continuous spectrum for Delone Hamiltonians, Duke Math. J., 131, 2 (2006), 203-217.

[19] D. LenZ AND I. Veselić, Hamiltonians on discrete structures: jumps of the integrated desity of states and uniform convergence, Math. Zeitschrift, 263 (2009), 813-835.

[20] P. MÜLleR AND C. RICHARD, Random colourings of aperiodic graphs: ergodic and spectral properties, arXiv:0709.0821v1, 2007.

[21] P. MÜLleR AND C. RichaRd, Ergodic properties of randomly coloured point sets, Canad. J. Math 65 (2013), 349-402.

[22] C. Rojas-Molina, Characterization of the Anderson metal-insulator transition for non ergodic operators and application, Ann. Henri Poincaré 13, 7 (2012), 1575-1611.

[23] C. Rojas-Molina, Etude mathématique des propriétés de transport des opérateurs de Schrödinger aléatoires avec structure quasi-cristalline, $\mathrm{PhD}$ thesis, Université Cergy-Pontoise, 2012.

[24] C. Rojas-Molina AND I. Veselić, Scale-free unique continuation estimates and applications to random Schrödinger operators, Comm. Math. Phys. 320 (2013), 245-274, DOI:10.1007/s00220-013$1683-4$.

[25] P. Stollmann, Caught by disorder: Bound states in random media, volume 20 of Progress in Mathematical Physics. Birkhäuser, Boston, 2001.

[26] A. SÜto, Schrödinger difference equation with deterministic ergodic potentials, Beyond Quasicrystals (Les Houches 1994), pages 481-549, 1995. 